\title{
Deformation, acoustic emission and ultrasound velocity during fatigue tests on paper
}

\author{
A. Miksic ${ }^{1, a}, J_{\text {Koivisto }}{ }^{1}$, E. Mykkänen ${ }^{1}$, J. Saarenpää $^{1}$ and M. Alava ${ }^{1}$, \\ K. Mustonen ${ }^{2}$, P. Karppinen ${ }^{2}$, T. Karppinen ${ }^{2}$ and E. Hæggström ${ }^{2}$ \\ ${ }^{1}$ Aalto University, Department of Applied Physics, P.O. Box 14100, FI-00076 Aalto, Finland \\ ${ }^{2}$ Division of Materials Physics, Faculty of Sciences, University of Helsinki, 00014 Helsinki, Finland
}

\begin{abstract}
We study the evolution of mechanical properties of paper samples during cyclic experiments. The issue is to look at the sample-to-sample variation, and we try to predict the number of loading cycles to failure. We used two concurrent methods to obtain the deformation: the strain was calculated from vertical displacement measured by laser interferometer sensor, as well as, computed by digital image correlation technique from pictures taken each $2 \mathrm{~s}$ by a camera. Acoustic emission of fracture was also recorded, and an active ultrasonic wave method using piezoelectric transducers is used to follow the viscoelastic behaviour of each sample. We found that a sharp final increase of different variables like deformation, strain rate and fluctuations, are signs of an imminent rupture of the paper. Moreover looking at the evolution of these quantities during the first cycle only is already an indicator about the lifetime of the sample.
\end{abstract}

\section{Introduction}

Paper is a viscoelastic material consisting of cellulose fibres. This disordered material is sensitive to lot of parameters like temperature or humidity [1], as cellulose fibres made from wood are hydrophilic. Paper properties are largely dependent on the fibres network. Paper is inhomogeneous and is effectively a two-phase composite due to the pore space inside the fibre network. The fracture of paper is as in all common materials, and continuum fracture mechanics is an important concept, but reaching a critical value for the external applied load is not the only condition for rupture. The load history has also an important effect [1-7]. Due to the quasi-two-dimensional nature of paper and the relevant length scales present it is a good test material for experimental fracture mechanics, apart from the fact that its mechanical behaviour is not quite understood. In this work, we consider fatigue fracture in the low-cycle limit, and in particular we overview our experiments where the main emphasis is on the issue to predict the failure of individual samples.

One of the most important scaling laws of time dependent fracture is the empirical Basquin's law for fatigue, which states that the lifetime of samples increases as a power law when the external load amplitude decreases [8-9]. This simple law is however an average and sample-to-sample variation may be quite important: in the results outlined below, a sample can break from $1^{\text {st }}$ to the $36^{\text {th }}$ cycle.

\footnotetext{
a e-mail : amiksic@cc.hut.fi
} 
In our work, we have studied various aspects of fatigue fracture, with an eye on prediction. To reach this goal we used diverse experimental methods like digital image correlation (DIC), acoustic emission recording and ultrasound wave propagation. The acoustic emission (AE) technique is widely used to study the fracture of paper [10-13] and other materials [14-17]. In parallel, wave propagation inside a heterogeneous sample offer a sensitive and non-invasive probe to follow the evolution of the elastic modulus [18-19].

In this article we will first review the mechanical tests performed on paper samples and the experimental techniques used. In the second part, we will present the experimental data collected about deformation by both used methods, as well as results from wave propagation and acoustic emission, and discuss their changes with time and number of cycles. Finally we will conclude that those diverse values and their evolution during first cycle or successive ones can give useful indications to predict the final break cycle.

\section{Experiments}

\subsection{Experimental set-up and samples}

We used $10 \times 5 \mathrm{~cm}^{2}$ samples from normal copy paper. In order to improve the grey scale contrast needed for the digital image correlation technique, samples are hatched with a drawing pencil and therefore the sample presents shadings on the side which is in front of the camera. The vertical axis of the sample is parallel with the cross-direction of paper. As the cellulose of the wood fibres is hydrophilic, paper is sensitive to humidity $[1,3]$; therefore the experimental set-up is enclosed in a chamber where temperature and humidity are stabilized $\left(\mathrm{T}=21.6^{\circ} \mathrm{C} \pm 0.3^{\circ} \mathrm{C}, \mathrm{RH}=50.5 \% \pm 0.7 \%\right)$.

The experimental setup is depicted in figure 1. The fixed frame is made of aluminium and a hook is fixed to the top crosshead. The sample is fixed between two steel clamps. Both clamps can move in translation, but can not turn as they are inserted in the rabbet of the lateral frame, that allows them only translate in vertical direction. The upper clamp holds on to the hook and so has a fixed height. The lower clamp is gripped onto the paper sample and holds a weight of $6,3 \mathrm{~kg}$, which applies a constant tensile force by hanging during loading phases. The lower clamp rests on two pistons during unloading. These pistons are moved by a system of compressed air controlled by computer and translate vertically on a run of $4 \mathrm{~cm}$. A LED lamp is used to illuminate the sample.

The test procedure consists of successive loading and unloading phases of $30 \mathrm{~s}$ each. The bottom clamp has a slow translation speed, corresponding to a strain rate around $6.3 \% . \mathrm{s}^{-1}$, in order to obtain a smooth stress application. The actual time under constant load is only $22 \mathrm{~s}$.

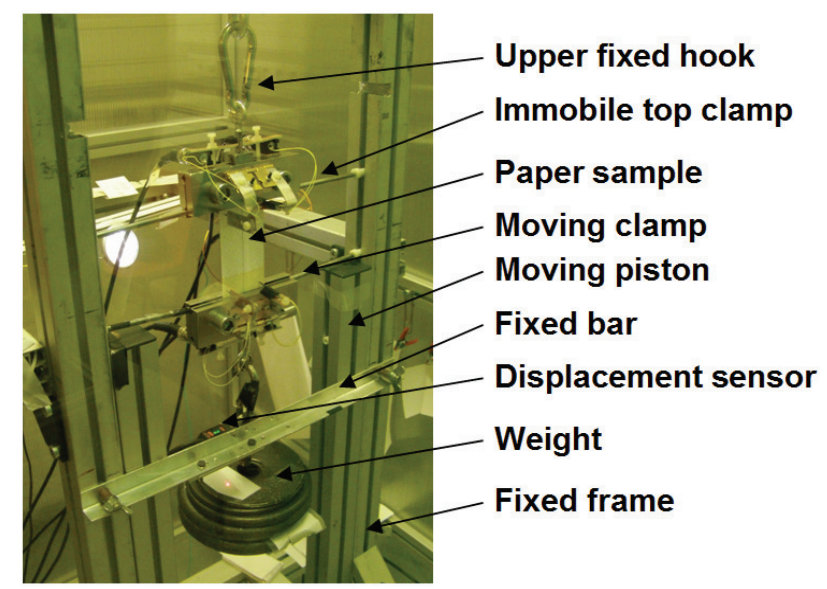

Fig. 1. Experimental set-up 


\subsection{Acquisition and treatment of experimental data}

\subsubsection{Deformation}

The deformation of the sample is obtained by two methods. First, the displacement of the bottom clamp is measured by a laser sensor, with an accuracy of $20 \mu \mathrm{m}$. On the other hand, we use the DIC technique [20-22]. A camera takes pictures of the sample each $2 \mathrm{~s}$ during the test, 1 pixel corresponding to $109 \mu \mathrm{m}$. The reference image is chosen as being from the beginning of the first fatigue cycle, i.e. the first picture where we can see that the sample is completely loaded. A regular network, defining squares of $10 x 10$ pixels ${ }^{2}$, is defined on the reference picture. A picture at time ' $t$ ' is then compared with this reference picture to obtain the displacement of each square, comparing grey scales of pictures. From these displacements, we can compute the averaged vertical (or horizontal) strain and average strain rate. DIC lets us focus on local deformation and videos made from successive pictures can show the evolution of deformation or fluctuations.

\subsubsection{Acoustic emission}

AE from the micro-fracturing of a paper sample is recorded by a physical acoustics piezoelectric transducer, attached to the upper clamp and gently pressed to the sample. While the transducers are generally fairly sensitive, direct contact to the paper was required to avoid need for excessive amplification. The acquisition chain contains a piezoelectric transducer, an amplifier, a 12-bit A/Dconverter and a PC with a four channel sample-and-hold type data acquisition hardware. In order to detect the high frequency AE events, high sampling rate is required. We used $312.5 \mathrm{kHz}$, which was the maximum supported by the A/D-converter. The first step towards analysing the AE signal is to detect and extract the actual events from the signal. This is done with a threshold algorithm which filters out any signal weaker than a specified limit. Acoustic emission energy is then calculated as the square integral of an event and AE time series consists of pairs of time and energy $\left(\mathrm{t}_{\mathrm{i}}, \mathrm{E}_{\mathrm{i}}\right)$. We are then interested in the distributions of energy and waiting times between two successive events.

\subsubsection{Ultrasound wave propagation}

A piezoelectric disc (diameter $5 \mathrm{~mm}$ ) was cemented onto a circuit board (LWD 30x10x2 $\mathrm{mm}^{3}$ ) with silver epoxy. Tungsten carbide needle was then glued on top of the piezoelectric disc. The electrical characteristics of the transducers are: $-6 \mathrm{~dB}$ bandwidth $150-350 \mathrm{kHz}$, central frequency $300 \mathrm{kHz}$, dielectric strength $1 \mathrm{kV}$. Four identical pieces were fabricated and fixed on the clamping mechanism. The coupling against sample surface was achieved by spring loading. As the transducers positioned on the top of the sample vibrate, they create shear and longitudinal waves which propagate into the paper sample. The waves are received by the two other transducer spikes on the bottom of the sample and the signals are recorded.

The signals from longitudinal and transverse waves are analyzed in the same manner. The reference signal is first defined as the signal corresponding to the beginning of first cycle (in the same way as the definition of reference picture for DIC). The initial time of flight is therefore measured for the reference signal by fitting 3rd degree polynomial curve on first peak; the time of flight at maximal amplitude is then read from this fit. Second, the changes in time of flight are defined by taking cross-correlation between the signal considered at time " $\mathrm{t}$ " and the reference one.

The wave speed is then calculated by the ratio of the travelled distance of wave divided by time of flight. The distance between transducer spikes is obtained directly from the reference picture taken by the camera. 


\section{Results and discussion}

We performed 40 identical tests. We observed that the final failure occurred between the first and the $36^{\text {th }}$ cycle. The average number of cycles to failure is 10 with a standard deviation of $70 \%$.

\subsection{Deformation from displacement measured by laser sensor}

The vertical strain $\varepsilon$ increases of few percents with the number of cycles $\mathrm{N}$ and with the time $t$ during each loading part because of creep, but decreases between two successive loads as recovery occurs during the unloading phase [23-24]. Thus the strain at the beginning of a creep phase is lower than at the end of the previous one (figure 2). Figure 2 shows the vertical deformation against time for each one of the successive loading part, in an experiment where the sample broke during the $7^{\text {th }}$ cycle.

The amount of deformation added during a creep phase is between $0.64 \%$ and $1.77 \%$ for the first cycle for all the experiments and tends to be bigger for samples with a short lifetime. This added strain decreases with the number of cycles and tends to a limit, between $0.14 \%$ and $0.25 \%$ for the longest tests. This quantity is however not enough to predict the rupture of the sample.

The curve of deformation versus time during each creep phase fits well with a logarithmic law $\varepsilon(\mathrm{t})=\mathrm{A} \cdot \log (\mathrm{t})+\mathrm{B}$. Values of B increase in a logarithmic manner with the number of cycles and are found to be from 0.0037 to 0.0148 . Values of A decreases with the number of cycles and tend toward a limit for long tests, as presented on figure 3. The logarithmic slope A seems to be higher for experiments where the sample breaks after a small number of cycles (inset in figure 3). The slope of the curve of strain versus time during the first cycle is therefore a good indication whether the rupture will occur rapidly or after a great number of cycles.
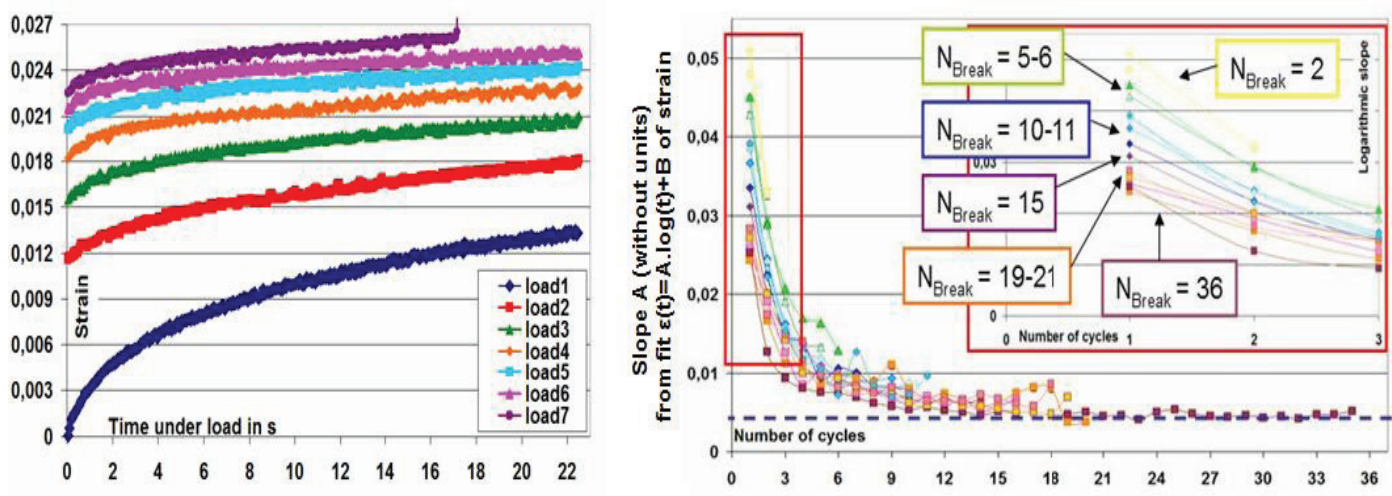

Fig. 2. Vertical strain (without unit or in $\mathrm{mm} / \mathrm{mm}$ ) vs. duration for each loading part

Fig. 3. Slope A (without unit) of logarithmic fit of $\varepsilon(t)$ vs. number of cycles. Inset is a zoom on the 3 first cycles

\subsection{Wave propagation}

The transverse waves propagate with velocities from $1620 \mathrm{~m} / \mathrm{s}$ to $1790 \mathrm{~m} / \mathrm{s}$ for all the different experiments and longitudinal waves travel faster, at $1880 \mathrm{~m} / \mathrm{s}-2070 \mathrm{~m} / \mathrm{s}$. Velocities of longitudinal wave are somewhat lower than expected [25]. Speeds of both waves present the same kind of evolution as the strain: they increase with time for each loading period and increase with the number of cycles too, and the magnitudes of velocity decrease between the end of a load phase and the beginning of the next one (figure 4). This first observation is not surprising as the travelling distance that waves should cover gets longer when vertical strain grows during loading. However we should notice that the time of flight decreases as well of $3-5 \%$ and has a predominant effect on this evolution. 
The relative change of velocity during creep for the longitudinal wave is between $7 \%$ and $3 \%$ for first cycles in all the experiments and diminishes with the number of cycles. The transverse wave's speed presents the same trend but its relative change is smaller. The ratio of longitudinal to transverse wave's velocities is found to be from 1.13 to 1.21 for the different samples and tends to rise with the number of cycles. The fact that longitudinal and transverse waves evolve differently highlights the anisotropy of the material [26]. No apparent correlation between these velocities ratios and the lifetime of samples was detected.

Secondly we observe that the longitudinal wave's velocity during the first creep phase seems to be correlated with the number of cycles at break: this speed is higher for tests with a larger number of cycles to rupture. This trend would be consistent with the previous results about strain. Theoretically, wave velocities are a function of the square root of elastic moduli divided by apparent volumetric mass of the sample [27]. Thus, the velocity variations detect the sample-to-sample variation of the initial elastic modulus, and its subsequent evolution during the experiment. A fast wave speed corresponds to a high elastic modulus and implies then a smaller strain for a given value of stress. Figure 4 depicts the square of the longitudinal wave speed averaged over the first load against the number of cycles at break; squared value of longitudinal waves velocity correspond to elastic modulus when considering apparent volumetric mass as unity.

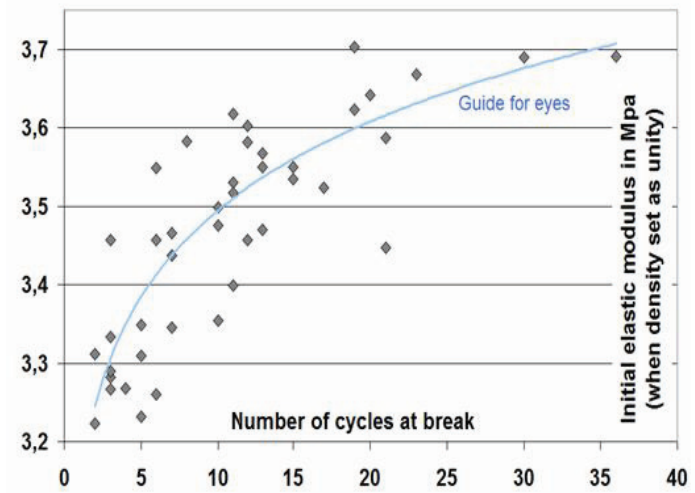

Fig. 4. Initial elastic moduli vs. number of cycles at break

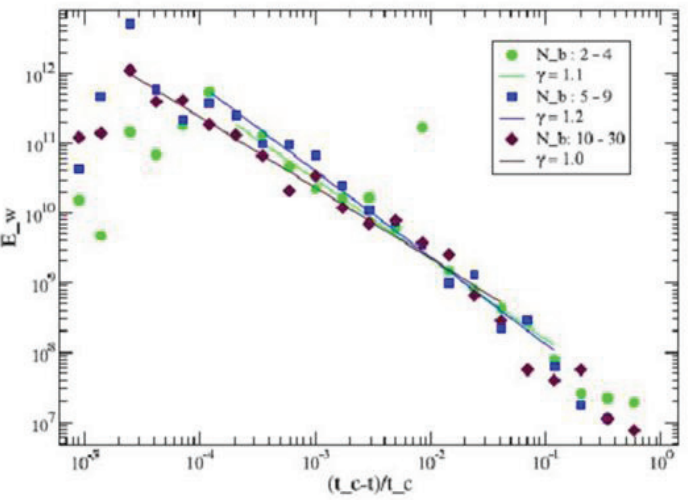

Fig. 5. AE energy vs. time normalised by lifetime

\subsection{Acoustic emissions}

Acoustic activity takes place mostly in the very last part of experiment, before the final break happens. The time during which acoustic emissions occur is very short and represents less than $10 \%$ of the lifetime. As in [28], figure 5 presents the repartition of AE energy against the change of time relatively to the time of break: $\left(\left(t_{c}-t\right) / t_{c}\right)$ where $t_{c}$ is the lifetime. This figure shows that most of AE energy is released during a short time before failure; for example, $E_{w}=10^{9}$ when $\left(\left(t_{c}-t\right) / t_{c}\right)=10^{-2}$, meaning that in a duration of $1 \%$ of the lifetime, there is $99.9 \%$ of the total energy released. The relationship found here between cumulative energy and relative time is a power law with an exponent between -1 to -1.2 and a divergence of the experimental data form this power law close to the rupture time is observed.

The distribution of waiting times and the distribution of energy for acoustic emission both exhibit a power law form $\mathrm{P}(\mathrm{t})=\mathrm{A} \cdot \mathrm{t}^{-\alpha}$ and $\mathrm{P}(\mathrm{E})=\mathrm{B} \cdot \mathrm{E}^{-\beta}$. The values of constants $\mathrm{A}$ are found from $0.50 .10^{7}$ to $1.8 .10^{7}$ and values for $\mathrm{B}$ from $4.2 \cdot 10^{-5}$ to $6.9 .10^{-5}$. Exponents values are $\alpha=1.11 \pm 0.05$ and $\beta=1.62 \pm 0.03$. The exponents vary slightly for the different samples and a light effect of the lifetime is observable, but no clear tendency between exponent values and lifetime can be concluded. We should point out that our exponent values are relatively close to $\alpha=1.3 \pm 0.2$ and $\beta=1.4 \pm 0.1$ for inter-event times and event energies respectively found in [11] for tensile experiment on paper. 


\subsection{Data from digital image correlation}

The averaged vertical strain is calculated over the sample surface, and is a bit smaller than the strain value obtained by the calculation from the laser sensor displacement. As from the mechanical data, deformation increases with time and number of cycles, and during unloading phases recovery happens (figure 6). Curves of deformation against time $\varepsilon(\mathrm{t})$ during creep cycles fit rather well with a logarithmic law but, especially for samples which break after a short number of cycles, a power law $\varepsilon(\mathrm{t})=\alpha_{1} \cdot \mathrm{t}^{\beta}$ seems to be more adapted, particularly for the first cycle. This observation is analogous with the evolution of deformation during a single creep test that presents three steps [29]: the primary creep at the beginning described by the Andrade's law $\varepsilon(t)=\alpha_{1} \cdot t^{\beta}$, with close to $1 / 3$, then a secondary creep phase presenting a logarithmic increase of deformation and finally the tertiary creep starting when the strain rate begins to rise again. Here the values of the exponent $\beta$ are found to spread from 0.27 to 0.78 for all the experiments, and $\alpha_{1}$ can be between $7.6 .10^{-4}$ and 5.4.10-3.

All the $\varepsilon(\mathrm{t})$ creep curves were then fitted with a logarithmic law $\mathrm{A} \cdot \log (\mathrm{t})+\mathrm{B}$ and compared with the results from mechanical data. Same trends are observed: the slope A from logarithmic fits decreases with the number of cycles and the slope during first cycle is clearly higher for tests in which the rupture of the sample occurs after a small number of cycles. Moreover by just comparing the first cycles for different tests, it seems that the strain is bigger for short tests and much smaller in a sample where the lifetime is important.

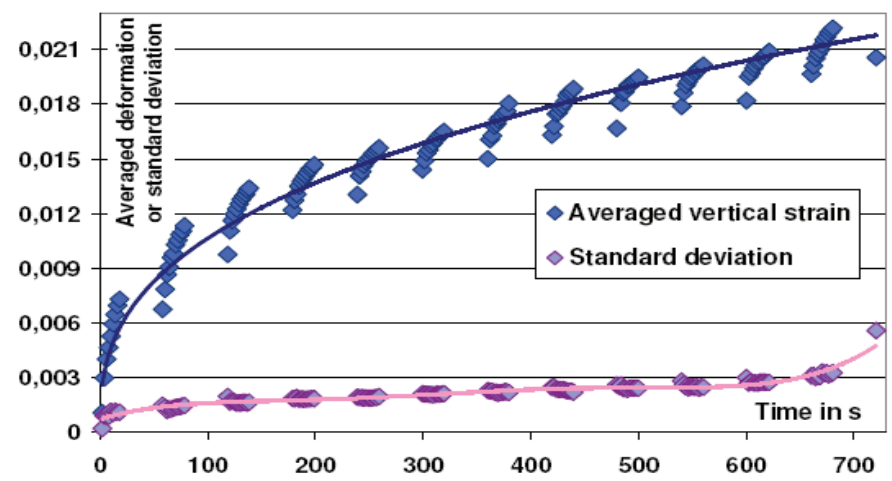

Fig. 6. Averaged vertical strain and its standard deviation vs. time

An interesting feature can be seen by looking at the standard deviation of the vertical strain, which corresponds to fluctuations of the strain around its averaged value. Like the averaged deformation, these fluctuations increase with the number of cycles, and a sharper growth is observed at the end of the test, a short time before that sample breaks. The relative changes with time, for each creep, in averaged vertical strain and in standard deviation decrease both with the number of cycles. The relative change of averaged strain is bigger than the relative change of fluctuation for most cycles, but it is the contrary for the last ones. Moreover we calculated the value of ratio "standard deviation/averaged strain" in percentage, and then averaged it for each creep phase. This ratio shows the strain fluctuations. This ratio decreases first with time and number of cycles, but rises strongly before the break. In addition, this ratio is higher for short lifetime samples, which means that the paper sample will break more rapidly when the amplitude of strain fluctuation is large.

In the evolution of the averaged strain rate we find that it decreases clearly with time for the first cycle, and then seems to be almost constant during each other cycle. Strain rate decreases first with the number of cycles, but again his quantity presents an increase before the rupture for every test. This is all the more visible when considering the average strain rate for each cycle (figure 7). A Monkman-Grant [30] type relationship exists between the time of minimum strain rate and the 
lifetime: $t_{\min }=0.925$. $t_{\text {break }}$ (figure 8 ). We should notice that the acoustic emission occurs after this $\mathrm{t}_{\min }$. The lifetime and time of minimum strain rate are calculated only during loading parts. But even if time including unloading phases is used, the same relationship is obtained. The ratio $t_{\min } / t_{\text {break }}$ is actually between 0.88 and 0.98 with an averaged value of 0.94 and standard deviation smaller than $4 \%$; this ratio is low for long lifetime samples and higher for samples that break after a small number of cycles.

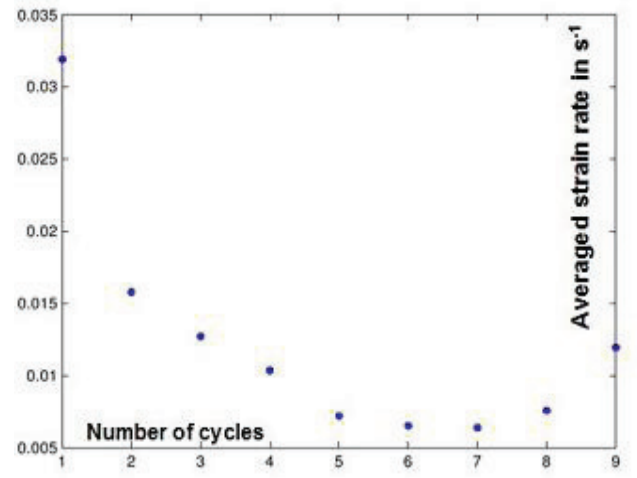

Fig. 7. Averaged strain rate vs. number of cycles

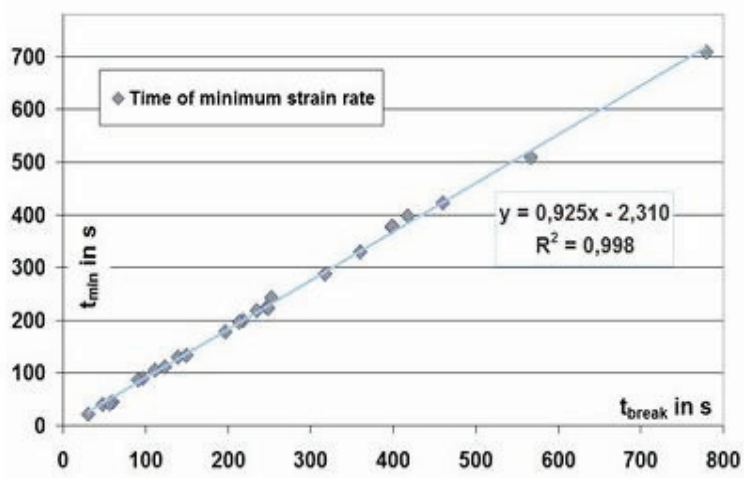

Fig. 8. Time of minimum strain rate vs. lifetime

DIC technique allows us to study the spatial aspects of strain over the sample. Strain is naturally smaller at the top of the sample than at the bottom of the sample (where force is applied). Before final failure, significant deformations are confined near the location where the final crack will propagate from. Spatial fluctuations in strain are also observed and localization appears with successive loading cycles. At the beginning of a test, during the first load phases, strain fluctuations are spread rather homogeneously on the whole sample area, but localization is seen with further cycling. Moreover, a correlation between fluctuations and lifetime seems to emerge: samples which break quickly present large strain fluctuations and the fluctuation amplitude could be more than two times smaller for long lifetime samples. Additionally the fluctuations are localised only close to the final crack for short lifetime sample. Just a few locations with higher fluctuations are seen for samples breaking after around 10 cycles while in contrast, for samples with a longer lifetime, large fluctuations are widely present over the sample.

\section{Conclusions}

We have analyzed cyclic creep experiments on paper using digital image correlation and acoustical techniques. We observed that acoustic emissions are clustered indicated by the power law behaviour of energy and waiting times; AE occurs predominantly right before break, during a time that is less than $10 \%$ of the lifetime. In the same manner, strain, strain rate, fluctuations of both of them and localisation of these fluctuation grow sharply just before failure. We found a Monkman-Grant type relationship $t_{\min }=0.925$. $_{\text {break }}$ for the strain rate.

We have shown that looking at the evolution of various data during first cycle only can already give interesting indications to predict the lifetime of the sample. The deformation during first creep tends to be larger for short lifetime samples and first cycle behaviour reflects the final lifetime. Second, a clear correlation appears between initial longitudinal wave speed (in-plane stiffness) and lifetime. In addition, short lifetime samples show strain fluctuations which are greater and more localised than in samples which last a long time before rupture. 


\section{References}

1. M. Alava, K. Niskanen, Progress in Physics 69, 669 (2006)

2. J. Bristow, P. Kolseth, Paper Structure and properties, Marcel Dekker, New-York (1986)

3. R. Mark, C. Habeger, J. Borch, M. Lyne (Eds.), Handbook of Physical Testing of Paper, $2^{\text {nd }}$ edition, 2 volumes, Marcel Dekker, New-York (2002)

4. D. Coffin, S. Boese, 3rd International Symposium on Moisture and Creep Effects on Paper and Containers, pp. 1-6 (1997)

5. D. Coffin, C. Habeger, IPST Technical Paper Series Number 821 (1999)

6. J. Koivisto, J. Rosti, M. Alava, Phys. Rev. Lett. 99, 145504 (2007)

7. A. Miksic \& al, submitted (2010)

8. S. Suresh, Fatigue of Materials, Cambridge University Press (Cambridge, 1991)

9. F. Kun, H. Carmona, J. Andrade Jr, H. Hermann, Phys. Rev. Lett. 100, 094301 (2008)

10. J. Rosti, X. Illa, J. Koivisto, M. Alava, J. of Phys. D: Appl. Phys. 42, 214103 (2009)

11. J. Rosti, J. Koivisto, M. Alava, J. Stat. Mech., P02016 (2010)

12. L. Salminen, J. Pulakka, J. Rosti, M. Alava, K. Niskanen, Europhys. Lett. 73, 55 (2006)

13. L. Salminen, A. Tolvanen, M. Alava, Phys. Rev. Lett. 89, 185503 (2002).

14. S. Muralidhara, B. Prasad, H. Eskandari, B. Karihaloo, Construction and Building Materials 24, 4, 479-486 (2010)

15. E. Landis, D. Whittaker, Engineering Mechanics 2000, 302, pp. 21-29 (2000)

16. J.M. Berthelot, J. Rhazi, Composites Science and Technology 37, 411(1990)

17. C. Hippsleya, D. Buttlea, C. Scruby, Acta Metallurgica 36, 441 (1988)

18. C. Habeger Jr, in: R. Mark, C. Habeger, J. Borch, M. Lyne, Handbook of Physical Testing of Paper, $2^{\text {nd }}$ edition, Marcel Dekker, New-York, Vol. 1, pp. 257-311 (2002)

19. X. Jia, C. Caroli, B. Velicky, Phys. Rev. Lett. 82, 9 (1999)

20. F. Hild, S. Roux, Strain 42, 69 (2006)

21. J. Kybic, M. Unser, IEEE Tran. Image Process. 12, 1427 (2003)

22. J. Considine, C. Scott, R. Gleisner, J. Zhu, 13th Fundamental Research Symposium, pp. 613-630 (2005)

23. F.A. Veer, J. Schönwälder, A. Heidweiller, N. Kuipers, 15th European Conference of Fracture Advanced, ECF15, pp. 1-8 (2004)

24. M. Mustalahti, J. Rosti, J. Koivisto, M. Alava, submitted (2010)

25. R. Wann, G. Baum, C. Habeger, IPC Technical Paper Series 72 (1979)

26. Y. Khidas, X. Jia, in: R. Garcia-Rojo, H.J. Hermann, S. McNamara (Eds.), Powders and Grains, Taylor \& Francis, London, pp. 309-312 (2005)

27. H. Pollard, Sound Waves in Solids, H.J. Goldsmith (Eds.), London (1977)

28. A. Guarino, S. Ciliberto, A. Garcimartın, R. Scorretti, Eur. Phys. J. 26, 141 (2002)

29. H. Nechad, A. Helmstetter, R. El Guerjouma, D. Sornette, Phys. Rev. Lett. 94, 045501 (2005)

30. F. Monkman, N. Grant, Proc. ASTM 56, 593 (1956) 